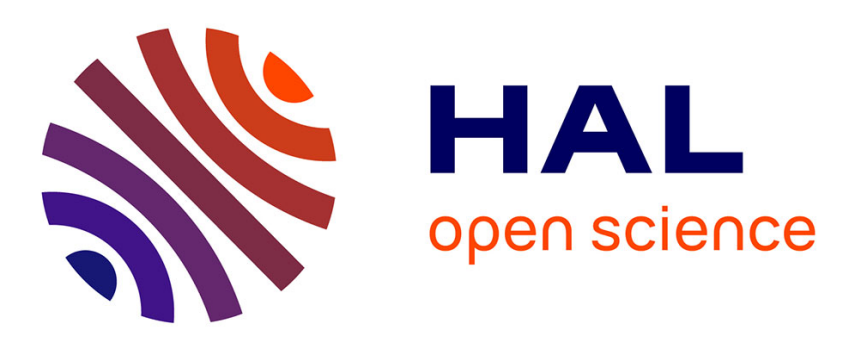

\title{
Sur l'observation et l'interprétation cinématique des phénomènes découverts par M. le Dr Zeeman
}

\author{
A. Cornu
}

\section{To cite this version:}

A. Cornu. Sur l'observation et l'interprétation cinématique des phénomènes découverts par M. le Dr Zeeman. J. Phys. Theor. Appl., 1897, 6 (1), pp.673-678. 10.1051/jphystap:018970060067300 . jpa-00240135

\section{HAL Id: jpa-00240135 https://hal.science/jpa-00240135}

Submitted on 1 Jan 1897

HAL is a multi-disciplinary open access archive for the deposit and dissemination of scientific research documents, whether they are published or not. The documents may come from teaching and research institutions in France or abroad, or from public or private research centers.
L'archive ouverte pluridisciplinaire HAL, est destinée au dépôt et à la diffusion de documents scientifiques de niveau recherche, publiés ou non, émanant des établissements d'enseignement et de recherche français ou étrangers, des laboratoires publics ou privés. 


\section{SUR L'OBSERVATION ET L'INTERPRÉTATION GINÉMATIQUE DES PHÉNOMÈNES DÉCOUVERTS PAR M. LE Dr ZEEMAN;}

Par M. A. GORNU.

Les phénomènes découverts par $\mathrm{M}$. le $\mathrm{D}^{\mathrm{r}}$ Zeeman, relatifs à l'action d'un champ magnétique sur les radiations émises par diverses sources lumineuses, ont donné lieu à quelques confusions qui me paraissent résulter de l'imperfection optique des modes d'observation. Les dispositifs suivants donnent une grande netteté à ces phénomènes et ne laissent aucun doute sur les conclusions définitives énoncées par l'auteur de la découverte ( $\left.{ }^{1}\right)$.

La source lumineuse est la flamme d'un chalumeau oxhydrique léchant un fragment d'amiante imbibé de chlorure de sodium fondu, ou bien l'étincelle d'induction jaillissant entre deux électrodes métalliques; elle est placée entre les deux pôles d'un électro-aimant produisant un champ magnétique intense.

Une fente verticale placée près de la source lumineuse, ou dans le plan d'une image focale de cette source, dirige le faisceau sur un réseau concave Rowland de 10 pieds de foyer, qui résout en raies brillantes le spectre de la source. Ce sont les raies spontanément renversables qui paraissent surtout déceler le phénomène.

Premier dispositif. - L'observation de l'une de ces raies se fait dans le plan focal d'un oculaire où l'on fixe une aiguille d'acier normalement aux raies spectrales. En arrière de l'oculaire est placé un prisme biréfringent de Wollaston $\left({ }^{2}\right)$ qui dédouble l'image de l'aiguille; le diamètre de cette aiguille, d'ailleurs légèrement conique, est choisi de manière que les deux images aient un bord commun. On obtient ainsi deux plages contiguës polarisées, l'une parallèlement, l'autre perpendiculairement aux raies spectrales.

$1^{\circ}$ Le faisceau est observé normalement aux lignes de force magnétiques.

Les deux pôles de l'électro-aimant 'bobines de Faraday, modèle

(1) Dr P. ZeEnan, Doublets and triplets in the spectrum produced by External Magnetic Forces (Philos. Magazine, for july 1897, p. วัว̆; for september 1897, p. 255). - Journ. de Phys., $3^{\mathrm{e}}$ série, VI, 143, 6 วั5.

(2) Un rhomboïde de spath pourrait, à la rigueur, remplacer le prisme de Wollaston: mais il y aurait quelques précautions à prendre pour éviter l'effet de parallaxe provenant de l'inégalité de distance des plans de vision des deux images.

J. de phys., $3^{\mathrm{e}}$ série, t. VI. (Décembre 1897.) 
ordinaire de Ruhmkorff), terminés par deux cônes arrondis, peuvent être rapprochés à 8 millimètres ou 10 millimètres, et l'on observe dans un plan perpendiculaire à la droite horizontale qui les joint.

On règle le prisme biréfringent de manière que les raies spectrales n'éprouvent aucune discontinuité sur la ligne de séparation des deux plages lorsque le champ magnétique est nul.

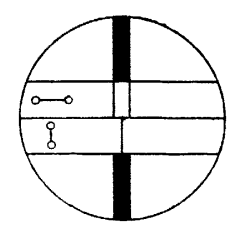

FIg. 1.

Dès qu'on excite l'électro-aimant, on voit la raie s'élargir; mais dans les deux plages polarisées l'aspect de la raie est modifié (fig. 1).

Dans la plage polarisée parallèlement aux lignes de force (ligne des pôles) la raie est dédoublée, c'est-à-dire présente une ligne sombre en son milieu; dans l'autre elle est, au contraire, amincie et se trouve exactement sur le prolongement de la ligne sombre préritée $\left({ }^{1}\right)$.

L'ínversion du pôle magnétique ne change en rien l'aspect du phénomène.

On en conclut que chaque raie simple primitive, non polarisée, est transformée en un triplet dont les composantes extérieures sont complètement polarisées parallèlement aux lignes de force et dont la composante intérieure est complètement polarisée dans un plan perpendiculaire. Le champ magnétique produit donc deux altérations de la période primitive, respectivement égales et de signe contraire, pour constituer les deux vibrations normales aux lignes de force, sans modifier celle de la vibration parallèle à ces lignes.

$2^{\circ}$ Le faisceau est observé parallèlement aux lignes de force.

L'une des armatures polaires est percée, suivant la ligne des pôles, pour livrer passage à la lumière suivant la direction des lignes de force.

Pour faire l'observation, on introduit entre l'oculaire et le prisme

(1) Lorsqu'on opère avec la lumière de la soude, chaque raie $D_{1}, D_{2}$ peut être plus ou moins renversée, c'est-à-dire plus ou moins dédoublée ; il en résulte une complication apparente, mais qui ne change pas le caractère essentiel du phénomène. 
biréfringent une lame de mica quart-d'onde dont les sections principales sont à $\mathbf{4 0}^{\circ}$ de celles du prisme. Dès que le champ magnétique est excité, on voit sur les deux plages la raie s'amincir et se briser sur la ligne de séparation ( $f g .2)$.

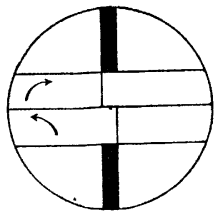

FIG. 2.

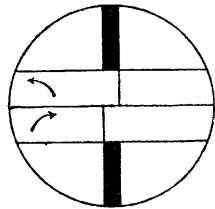

FIs. 3 .

Si l'on tourne la lame quart-d'onde d'un angle droit, la brisure se fait en sens inverse ( $f g .3$ ).

L'inversion des pôles intervertit le sens de la brisure. On reconnaît en outre que le milieu des deux raies ainsi produites occupe sensiblement la position de la raie primitive : les deux altérations de la période sont donc égales et de signe contraire.

On rond le phénomène encore plus visible en fixant côte à côte, sur une même glace, deux lames quart-d'onde à sections homologues rectangulaires; un petit mouvement alternatif de translation imprimé à ces lames donne alternativement les deux apparences précitées. Un rythme convenable accroît encore la sensibilité de la méthode; car, lorsque l'œil fixe la raie de l'une des plages, lo déplacement relatif de la raie correspondante de l'autre plage se trouve physiologiquement doublé.

Ces apparences prouvent que l'action du champ magnétique dédouble chaque radiation en deux faisceaux polarisés circulairement de sens inverse, le renversement des pôles renversant le sens de la rotation des vibrations circulaires.

En déterminant le sens de la rotation de chacun de ces faisceaux ( $\left.{ }^{1}\right)$, on parvient à résumer l'ensemble des résultats dans l'énoncé très simple que nous donnons plus loin.

Second dispositif. - Au lieu d'un long prisme biréfringent de

(1) Cette détermination correcte n'est pas aussi facile qu'on pourrait le supposer; on risque de commettre des erreurs dont $\mathrm{M}$. le $\mathrm{D}^{\mathrm{r}}$ Zeeman lui-même a connu le danger (loc. cit., p. 58). Dans une prochaine note je me propose d'indiquer diverses méthodes optiques qui permettent d'effectuer pratiquement et de vérifier ce genre de détermination. 
Wollaston (nécessaire pour obtenir deux plages suffisamment larges) on peut utiliser un simple prisme de Nicol; le double champ s'obtient alors avec des lames de mica convenablement choisies et orientées.

$1^{\circ}$ Faisceau observé normalement aux lignes de force.

Dans le plan de l'oculaire on place deux lames demi-onde, réglées sur la réfrangibilité de la raie brillante employée $\left({ }^{1}\right)$.

La lame supérieure a ses sections principales parallèles ou perpendiculaires à la direction des raies spectrales, la lame inférieure à $\pm 4 \breve{\partial}^{\circ}$ de ces raies. Si la section principale du prisme de Nicol est parallèle ou perpendiculaire à cette direction, on obtient la même apparence $(f g .1)$ qu'avec le premier dispositif, parce que la seconde lame demi-onde fait tourner d'un angle droit les plans de polarisation des faisceaux qu'elle transmet.

$2^{\circ}$ F'aisceau observé parallèlement aux lignes de force.

Dans le plan focal de l'oculaire on place deux lames quart-d'onde; la lame supérieure a ses sections principales à $+4 \check{\partial}^{\circ}$, l'inférieure à - $\measuredangle \widetilde{0}^{\circ}$ de la direction des raies; le prisme de Nicol étant réglé comme précédemment, on obtient la même apparence ( $f g$. . 2) qu'avec le premier dispositif.

Si l'on veut produire le balancement rythmé, on dispose un second système en ordre inverse qu'on fixe à côté du premier dans l'ordre figuré comme il suit :

\begin{tabular}{l|l}
\hline$+45^{\circ}$ & $-45^{\circ}$ \\
\hline$-45^{\circ}$ & $+45^{\circ}$ \\
\hline
\end{tabular}

La translation alternative de ce système à droite et à gauche produit l'inversion et la duplication apparente si favorable à l'observation du phénomène.

Interprétation cinénatique des phécomènes. - L'ensemble des phénomènes peut se résumer en un énoncé conforme aux règles de Fresnel et d'Ampère.

Voici d'abord les règles auxquelles il est fait allusion:

(1) Le réglage correct de la biréfringence des lames de mica exige aussi des soins particuliers qui seront l'objet de quelques développements dans l’Appendice annoncé. 
$1^{\circ}$ Un faisceau de lumière ordinaire est la superposition de deux faisceaux indépendants égaux en intensité et polarisés à angle droit (FresneL);

20 Un faisceau polarisé rectilignement est la superposition de deux faisceaux égaux en intensité et polarisés circulairement en sens inverse (FrESNEL) ;

$3^{\circ}$ Une ligne de force magnétique équivaut à l'axe d'un solénoïde dont le pôle austral est à la gauche du courant (AMpène).

L'action du champ magnétique sur l'émission d'une radiation tend $\dot{a}$ décomposer les composantes rectilignes vibratoires susceptibles de se propager par ondes suivanl des vibrations circulaires parallèles aux courants du solénoïde.

Les vibrations qui tournent dans le sens du courant du solénoïde sont accélérées, celles qui tournent en sens inverse sont retardées.

On reconnaît immédiatement le doublet observé dans le sens des lignes de force.

Dans le sens perpendiculaire à ces lignes cet énoncé montre que la composante parallèle aux lignes de force (onde polarisée perpendiculairement à cette direction) est inaltérée: c'est la raie médiane du triplet; les deux raies extérieures polarisées à angle droit de celle-ci sont plus difficiles à reconnaître. Cependant on aperçoit géométriquement leur existence. En effet, c'cst ce qui subsiste des deux vibrations circulaires, l'une accélérée, l'autre retardée, en lesquelles le champ magnétique dédoublait la composante normale aux lignes de force; il y a extinction ou compensation mutuelle des deux composantes longitudinales qui ne peuvent pas se propager (les deux ondes polarisées rectilignement sont produites par les vibrations circulaires de sens inverse, vues de tranche).

Cette interprétation purement cinématique, quoique un peu superficielle, montre que le phénomène découvert par $\mathrm{M}$. le $\mathrm{D}^{\mathrm{r}}$ Zeeman peut être expliqué par des considérations tout à fait indépendantes des idées électrochimiques de M. le professeur Lorentz, qui en sont l'origine, et se rapproche beaucoup des théories tourbillonnaires récemment remises en honneur.

Elle montre, en outre, la différence essentielle qui existe entre ce phénomène et celui du pouvoir rotatoire magnétique découvert par Faraday.

L'action du champ magnétique sur les sources où les ondes sont pour ainsi dire à l'état naissant s'exerce sur la période vibratoire, 
tandis que, dans l'expérience de Faraday, elle s'exerce sur la vitesse de propagation d'ondes lumineuses ayant déjà acquis leur régime permanent.

Je me suis assuré, avec les mêmes dispositifs, que la rotation magnétique du plan de polarisation n'est accompagnée d'aucune variation sensible de la période vibratoire de la lumière monochromatique employée, tandis que j'ai démontré autrefois (1) que la vitesse de propagation des deux ondes circulaires est modifiée: l'une est accélérée, l'autre est retardée de quantités sensiblement égales dans le sens correspondant à la règle d'Ampère. 